\title{
Genève, le temps d'un serment
}

par Marinette Ummel

\section{RÉsumé}

La crise de l'éthique médicale provoquée par les atrocités commises, lors de la deuxième guerre mondiale, par les médecins nazis, suscita l'élaboration, en 1948, d'une version, adaptée au vingtième siècle, de l'antique Serment d'Hippocrate: le Serment de Genève. L'importante notion d'Humanité, qui apparaît dans celui-ci - sans y être clairement définie - pour qualifier le devoir du médecin, sera la base d'une réflexion sur les limites que ce concept peut se voir attribuer en médecine.

Formulation moderne du Serment d'Hippocrate, c'est bien le Serment de Genève qui se cache derrière ce titre quelque peu énigmatique.

Il a été adopté en 1948, lors d'un bref séjour de la toute jeune Association Médicale Mondiale (AMM) pour sa deuxième assemblée générale, à Genève. S'il est, actuellement, quelque peu tombé en désuétude, il représente encore une référence en matière d'éthique médicale.

A la suite de la révélation des atrocités commises lors de la deuxième guerre mondiale, souvent justifiées scientifiquement par les médecins nazis, la profession médicale s'est trouvée gravement remise en cause.

Lors du procès de Nuremberg, ${ }^{1}$ pendant lequel ces médecins furent jugés, le Serment d'Hippocrate fut, certes, reconnu comme la seule référence d'éthique médicale existante à ce moment-là, mais sa valeur et son rôle furent, toutefois, très discutés. Pour certains, l'universalité des principes qu'il énonçait le rendait toujours applicable, d'autres le remettaient en cause parce qu'ils le trouvaient inadapté aux problèmes spécifiques de la médecine de cette époque, notamment à ceux de la recherche médicale.

Le tribunal, avant de rendre son jugement, en août 1947, énonça dix nouveaux principes qui fixaient le cadre dans lequel des expérimentations humaines pouvaient se pratiquer en médecine. Ces règles, qui portent le nom de Code de Nuremberg, ne concernent que l'expérimentation humaine et s'inspirent, en majeure partie, des principes éthiques qui étaient reconnus par le corps médical de l'époque, aux Etats-Unis, dans ce domaine. L'obliga- 
tion pour le chercheur d'obtenir dans tous les cas le consentement des personnes qui participent à la recherche médicale, constitue le point central de ce texte.

Les prises de positions de la communauté médicale internationale, par le truchement de l'AMM, diffèrent, dans un premier temps, de celles qu'avaient adoptées le tribunal de Nuremberg.

La constitution de l'AMM fut officiellement ratifiée à Paris ${ }^{2}$, le 18 septembre 1947, par les délégués des associations médicales nationales de quarante-huit pays. Cette nouvelle organisation remplaçait l'Association Professionnelle Internationale des Médecins qui avait suspendu ses activités pendant la guerre.

Les objectifs de l'AMM, consistaient à promouvoir la paix et la santé dans le monde entier en resserrant les liens entre les médecins tant au niveau personnel que scientifique. L'Association visait aussi à constituer pour les médecins un porte-parole efficace au niveau international. Aujourd'hui, elle est surtout connue pour ses déclarations de principes concernant différents problèmes de la médecine. Les plus connues sont le Serment de Genève et la Déclaration d'Helsinki sur la recherche médicale.

L'AMM se réunit au moins une fois tous les deux ans en assemblée générale $^{3}$. Son secrétariat, qui se trouvait d'abord à New-York, s'est installé depuis 1974, à Ferney-Voltaire.

Lors de sa première assemblée générale ${ }^{4}$, qui se tint à Paris après sa création officielle, l'AMM condamna sévèrement les crimes commis par les médecins nazis pendant la deuxième guerre mondiale. Elle adopta une formule de serment qui devait être prononcée par les nouveaux médecins praticiens. Ce texte rappelait notamment le respect de la dignité humaine pour tous les patients, quelle que soit leur situation politique, raciale et religieuse.

L'AMM demandait aussi que le syndicat des médecins allemands fasse une déclaration publique qui comportait la reconnaissance des actes de cruauté commis pendant la guerre, la condamnation des organisations médicales qui n'y avaient pas réagi et l'exclusion des criminels par les associations médicales.

L'assemblée générale de l'année suivante, en 1948, avait été prévue à Prague. Toutefois, en raison des graves troubles politiques que subit ce pays et qui avaient occasionné la dissolution de la société médicale tchèque, l'AMM ne put se rendre dans cette ville.

Genève fut alors choisie ${ }^{5}$ pour accueillir cette réunion, notamment parce 


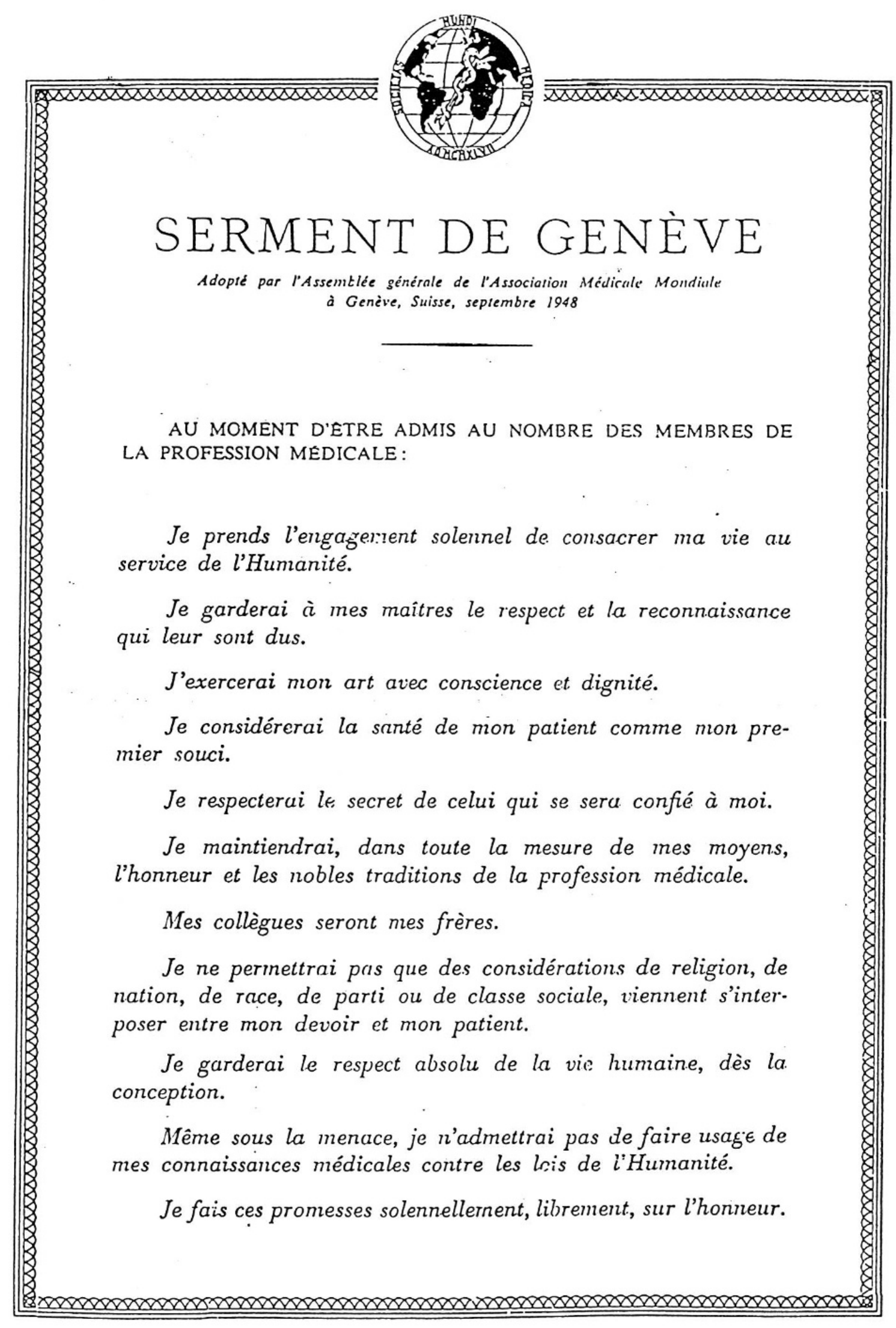


qu'elle était le siège de plusieurs organisations internationales et de la Croix Rouge.

En lisant la Tribune ${ }^{6}$ et le Journal ${ }^{7}$ de Genève de l'époque, on apprend que le 8 septembre 1948, au matin, les délégués de l'AMM furent reçus dans la salle du Grand Conseil genevois. Le Conseiller d'Etat Picot, également président du Conseil National, les salua au nom des autorités fédérales, cantonales et municipales. L'après-midi, les participants visitèrent l'Agence centrale des prisonniers de guerre; ils furent conviés, le soir, à une réception offerte par le Conseil d'Etat et le Conseil administratif genevois au Palais Eynard. La Fédération des Médecins suisses offrit le dîner du 9 septembre et l'assemblée se termina le 12 septembre, à Chamonix, avec le syndicat médical de Haute-Savoie.

Les délégués ne se contentèrent toutefois pas de profiter des charmes hospitaliers de Genève, mais discutèrent de sujets moins réjouissants. En effet, il fallait rétablir la situation de l'éthique médicale bafouée pendant la guerre. Les délégués de l'AMM décidèrent de remettre en vigueur les principes fondamentaux du Serment d'Hippocrate. C'est pourquoi, ils adoptèrent une nouvelle version de ce texte, adaptée au vingtième siècle. Les médecins liraient cette formule à leur remise de diplôme.

Sur la proposition de deux délégués, anglais et danois, le nom de Genève fut choisi pour dénommer cette nouvelle version du Serment d'Hippocrate qui devint ainsi le Serment ou la Déclaration de Genève.

L'AMM nomma encore le Docteur Jean Maystre, président de l'Association des médecins de Genève, comme délégué auprès des organisations internationales, et s'engagea à soutenir dans la mesure de ses moyens l'ASEM (association suisse, dont le siège se trouvait à Genève, qui s'occupait de l'aide aux enfants des médecins victimes de la guerre).

L'adoption du Serment de Genève et les décisions prises par l'AMM furent bien accueillies par la presse et les médecins genevois, comme en témoignent la Tribune de Genève ${ }^{8}$ et le Bulletin professionnel des médecins suisses ${ }^{9}$.

Le Serment de Genève ne constitue pas seulement une version du Serment d'Hippocrate, modernisée et dépouillée des références mythologiques antiques. Il est indéniablement une réponse spécifique aux interrogations pendantes, quant au rôle de la médecine, qu'avaient provoquées les horreurs commises par les médecins nazis, pendant la deuxième guerre mondiale.

Le Serment de Genève fait apparaître le terme d'Humanité, à deux reprises, jouant sur deux de ses acceptions: l'Humanité comprise soit comme l'ensemble des êtres humains soit comme le caractère de ce qui est humain. Ce 
concept constitue peut-être la différence la plus importante entre le Serment de Genève et celui d'Hippocrate. Il implique un double élargissement du rôle du médecin. D'une part, son devoir ne s'arrête plus à ses patients en temps qu'individus, mais concerne l'Humanité entière. D'autre part, le médecin devient responsable, non seulement de l'efficacité des soins qu'il prodigue, mais également de leur Humanité.

Mireille Delmas-Marty ${ }^{10}$, présidente de la Commission Justice pénale et Droits de l'homme du Ministère de la justice français, affirme:

«Il faudrait enraciner davantage l'idée que l'humanité implique nécessairement la dignité $e t$ la vie, donc que s'il y a vie il doit y avoir également respect de la dignité».

Cette notion d'Humanité, impliquant la dignité de l'Homme peut se révéler, dans certaines situations, en contradiction avec ces deux autres exigences du Serment de Genève que sont «le respect absolu de la vie» et «la santé comme premier souci du médecin». Ces discordances semblent peut-être plus manifestes à notre époque de libéralisation de l'avortement et de progression très rapide des sciences médicales qui rend les limites entre la vie et la mort de plus en plus floues.

De même, on peut se demander pourquoi l'AMM n'a pas, dans le Serment de Genève, mentionné spécifiquement l'expérimentation humaine ou même fait allusion au Code de Nuremberg. Il est vrai que toute une frange de chercheurs trouvait ce dernier trop rigide et que l'AMM, loin de se désintéresser du sujet, a préféré se donner le temps d'une réflexion approfondie avant de rédiger, en 1964, la première version de la Déclaration d'Helsinki. De plus, le fait que le Serment de Genève mentionne le devoir, pour le médecin, de servir l'Humanité, implique, certes de manière voilée, mais plus clairement que ne le faisait le Serment d'Hippocrate, la recherche de nouveaux traitements. La pratique de l'expérimentation humaine, si elle vise bien l'amélioration de la santé de certains individus, ne va pas sans causer des désavantages à d'autres. Si elle semble envisagée dans le Serment de Genève, elle n'en reste pas moins problématique, puisque la quantité et la nature des inconvénients admissibles en vue d'un certain bénéfice sont définis et limités par le caractère «humain» - critère non défini concrètement dans le Serment - qu'elles doivent satisfaire.

Loin d'être seulement paralysants, ces paradoxes ou ces imprécisions illustrent bien les points de tension que rencontre le médecin dans l'exercice de sa profession.

La deuxième moitié du vingtième siècle a bien montré que les directives 
éthiques élaborées pour un problème particulier de la médecine doivent inévitablement être modifiées suivant l'avance des progrès scientifiques en ce domaine. Cela implique que les principes d'éthique ne sont plus forcément universels.

Le Serment de Genève n'est certes pas spécifique d'un domaine particulier de la médecine, toutefois son évolution démontrera qu'il n'est plus considéré, dans son intégralité, comme universel dans le temps.

En effet, si le Serment d'Hippocrate a perduré plus de deux millénaires, sans modification, l'AMM a déjà modifié le Serment de Genève à deux reprises ${ }^{11}$, en 1968 et en 1983.

De même la Déclaration d'Helsinki concernant la recherche biomédicale a été modifiée à trois reprises pour trouver les limites adéquates dans lesquelles peut se pratiquer une expérimentation réellement «humaine», c'est-à-dire, impliquant des sujets humains en respectant leur dignité, et servant à améliorer la santé ou le bien-être du sujet d'expérimentation ou des autres êtres humains.

L'évolution de ce texte et la nature de ses modifications se montrent particulièrement révélatrices.

En effet, considérant que la conscience seule du médecin ou du chercheur ne suffisait pas pour garantir toujours l'application des normes, l'AMM, en s'inspirant de l'expérience de quelques associations médicales nationales, recommanda, dans la deuxième version de la Déclaration d'Helsinki, la création de comités d'experts, multidisciplinaires, chargés d'examiner si les protocoles de recherche satisfaisaient aux exigences éthiques fixées dans les directives.

Des comités analogues ont aussi été créés, depuis le début des années 1980, pour examiner des situations cliniques, éthiquement problématiques.

Catherine Labrusse-Riou ${ }^{12}$, professeur de droit et membre du Comité National d'Ethique français, écrit:

«Ce qui manque, dramatiquement, à nos pensées, c'est le fondement de la limite».

Il semble bien que la société en général et la médecine en particulier cherchent les limites dans lesquelles les grands principes éthiques qui les fondent, tels que le respect de la vie et la dignité par exemple, peuvent se réaliser. Et cette recherche se caractérise, actuellement, par le fait qu'elle ne concerne plus seulement le contenu des principes, mais aussi la procédure par laquelle on peut trouver la meilleure manière de les rendre valables ou de les appliquer. 
Les comités d'éthique multidisciplinaires qui essaient en médecine de rassembler et d'impliquer des représentants de toutes les parties concernées par un problème ne remplacent pas, certes, la conscience du médecin et comportent des failles - preuves en sont les critiques dont ils sont l'objet et les abus encore commis actuellement en médecine - mais ils permettent, peut-être, de trouver, parfois, une meilleure adéquation de l'action du médecin aux limites particulières et propres à chaque patient ou à chaque personne participant à une recherche médicale ${ }^{13}$.

Même si, actuellement, le Serment de Genève n'est pas systématiquement prêté par les médecins, ses principes restent valables dans les limites que les représentants du genre humain, médecins ou non, voudront bien reconnaître à l'Humanité. Car les meilleures règles garantissant le respect de l'Humanité ne protègent pas ceux qui sont considérés comme n'en faisant pas partie. 
1 Bayle François, Croix gammée contre caducée, Paris, 1950, p. 1415-1520.

Ambroselli Claire, L'éthique médicale, Paris 1988.

2 Editorial, World Medical Association, in British Medical Journal sept. 27, 1947, p. 498-500.

3 Wynen André, Qu'est-ce que l'AMM ? in Publications de l'AMM, novembre 1980.

4 Editorial: World Medical Association, in Journal of the American Medical Association 135/ 8, 1947, p. 516-517.

5 Editorial: World Medical Association, in British Medical Journal sept. 25, 1948, p. 605-606. Editorial: World Medical Association, in Journal of the American Medical Association 138/ 6,1948, p. 435-436.

Editorial: World Medical Association, in Journal of American Medical Association 138/5, 1948, p. 366.

6 La Tribune de Genève, A l'assemblée générale de l'Association Médicale Mondiale, mercredi 8 et jeudi 9 septembre 1948, p. 16.

La Tribune de Genève, Une réception au Palais Eynard, vendredi 10 septembre, 1948, p. 3.

7 Le Journal de Genève, L'association Médicale commence ses travaux, mercredi 8 et jeudi 9 septembre, 1948, p. 10.

8 La Tribune de Genève, Après l'assemblée générale de l'Association Médicale Mondiale, lundi 20 septembre 1948 p. 3.

9 Mégevand Emile-C., $75^{\circ}$ assemblée générale de la Société Médicale de la Suisse romande; discours du président, in Bulletin professionnel des médecins suisses 41, 1948, p.512-513.

Maystre Jean, Discours officiel, in Bulletin professionnel des médecins suisses 47, 1948, p. 577-579.

10 Entretien avec Mireille Delmas-Marty, L'homme des droits de l'homme n'est pas celui des biologistes, in Esprit, novembre 1989, p.116-123.

11 Les changements concernent le secret médical, qui doit être maintenu «même après la mort du patient», et le respect absolu de la vie, qui s'exerce non plus dès «la conception» mais dès «son commencement».

12 Labrusse-Riou Catherine, L'homme à vif: biotechnologies et droits de l'homme, in Esprit, novembre 1989 , p. 60-70.

13 Cf. aussi Ummel Marinette, 1948, le Serment de Genève et la Déclaration des Droits de l'homme, quelles limites?, in Equinoxe 4, 1990, p. 109-112. 


\section{Summary}

\section{The Oath of Geneva}

The atrocities of Nazi doctors as revealed by the Nuremberg trial caused the World Medical Association in 1948 to formulate a modernized version of the Hippocratic Oath. The author discusses particularly the notion of Humanity, newly introduced in this so-called Oath of Geneva: contradictions may arise between medical interests and criteria of humanity, which make it necessary to draw limits.

\section{Zusammenfassung}

\section{Das Genfer Ärztegelöbnis}

Die Greueltaten nationalsozialistischer Ärzte, die der Nürnberger Prozess ans Licht brachte, veranlassten den Welt-Ärzte-Bund, 1948 eine modernisierte Version des Hippokratischen Eides auszuarbeiten, den sog. Serment de Genève. Die Autorin geht besonders auf den darin als Novum enthaltenen Begriff der Humanité ein, der die Menschheit ebenso wie die Menschlichkeit bezeichnet: Spannungen zwischen medizinischen Zielen und Kriterien der Menschlichkeit können auftreten und Grenzziehungen nötig machen.

Marinette Ummel

Institut universitaire de Médecine légale

Centre médical universitaire

1211 Genève 4 